\title{
Selection with inbreeding control in simulated young bull schemes for local dairy cattle breeds
}

\author{
G. Gandini, ${ }^{11}$ A. Stella, $\uparrow$ M. Del Corvo, $†$ and G. B. Jansen $\neq$ \\ *Department of Veterinary Science and Public Health, University of Milan, 20133 Milan, Italy \\ †IBBA, National Research Council, 26800 Lodi, Italy \\ fDekoppel Consulting, 10010 Chiaverano, Italy
}

\section{ABSTRACT}

Local breeds are rarely subject to modern selection techniques; however, selection programs will be required if local breeds are to remain a viable livelihood option for farmers. Selection in small populations needs to take into account accurate inbreeding control. Optimum contribution selection (OCS) is efficient in controlling inbreeding and maximizes genetic gain. The current paper investigates genetic progress in simulated dairy cattle populations from 500 to 6,000 cows undergoing young bull selection schemes with OCS compared with truncation selection (TS) at an annual inbreeding rate of 0.003. Selection is carried out for a dairy trait with a base heritability of 0.3 . A young bull selection scheme was used because of its simplicity in implementation. With TS, annual genetic gain from 0.111 standard deviation units with 500 cows increases rapidly to 0.145 standard deviation units with 4,000 cows. Then, genetic gain increases more slowly up to 6,000 cows. At the same inbreeding rate, OCS produces higher genetic progress than TS. Differences in genetic gain between OCS and TS vary from to 2 to $6.3 \%$. Genetic gain is also improved by increasing the number of years that males can be used as sires of sires. When comparing OCS versus TS at different heritabilities, we observe an advantage of OCS only at high heritability, up to $8 \%$ with heritability of 0.9 . By increasing the constraint on inbreeding, the difference of genetic gain between the 2 selection methods increases in favor of OCS, and the advantage at the inbreeding rate of 0.001 per generation is 6 times more than at the inbreeding rate of 0.003 . Opportunities exist for selection even in dairy cattle populations of a few hundred females. In any case, selection in local breeds will most often require specific investments in infrastructure and manpower, including systems for accurate data recording and selection skills and the presence of artificial insemination and breeders organizations. A cost-benefit analysis is therefore advis-

Received June 26, 2013.

Accepted November 27, 2013.

${ }^{1}$ Corresponding author: gustavo.gandini@unimi.it able before considering the implementation of selection schemes in local dairy cattle breeds.

Key words: inbreeding control, selection, local dairy cattle

\section{INTRODUCTION}

In 2007, the international community adopted the first Global Plan of Action for Animal Genetic Resources (FAO, 2007), which aimed to combat the ongoing erosion of farm animal genetic diversity and use sustainable animal genetic resources. Maintenance of local breed diversity is a widely accepted conservation goal (FAO, 2007) because it contributes substantially to the world farm animal genetic diversity. Many local breeds supply important ecosystem services and are associated to specific landscapes and rural cultural diversity, which are additional strong motivations for their maintenance (Gandini and Villa, 2003). Diversity of local breeds also increases the ability of communities to maintain their livelihoods in marginal areas (FAO, 2007). Incentive payments to farmers to compensate for the lower profitability of local breeds compared with substituting these breeds with more profitable exotic breeds have been often suggested and are currently in use within the European Community (European Union, 2005 ) to halt local breed erosion. However, public incentives are unlikely to be stable in the long-term, and therefore the need to have a diversity of profitable, selfsustaining, local breeds exists.

Breeds are dynamic entities, undergoing continuous genetic changes in response to both selection by farmers and environmental factors. Local breeds are rarely subject to index selection because of their small population sizes and poor technical support; however, advanced selection programs will undoubtedly be required if local breeds are to remain a viable livelihood option for farmers (FAO, 2007). Selection in local breeds might be used to avoid reduced performance in fitness traits and in traits with high market value, or more generally to improve animals for specific production traits. Because of the usually small population size of local breeds, their selection schemes have a particular need to con- 
trol inbreeding and must be based on a comprehensive evaluation of traits proposed for genetic improvement to guarantee the maintenance of within- and amongbreed genetic diversity.

Different strategies have been proposed to maintain genetic diversity in selection programs (Fernandez et al., 2011). The most sophisticated strategy is selection with optimal contributions (OC; Meuwissen, 1997; Grundy et al., 1998), which maximizes genetic gain with restriction on inbreeding rate. The OC method has been tested in large livestock populations by comparing optimized retrospective selection with the observed values in one step of selection with constraints on inbreeding (Avendaño et al., 2003; Kearney et al., 2004; Koenig and Simianer, 2006; Sørensen et al., 2008). Optimal contributions selection has rarely been simulated in realistic populations across a certain number of generations (Hinrichs and Meuwissen, 2011; Gourdine et al., 2012). In general, the benefits of using OC relative to truncation selection (TS) has been mainly shown in populations undergoing simplified selection schemes, and a need exists to analyze limits and benefits under different conditions that can be encountered in field conditions.

The objective of the current paper is use simulation to investigate the genetic progress that can be achieved in small dairy cattle populations undergoing a young bull selection scheme with inbreeding control. We considered a young bull scheme because it could be easily implemented in local breeds, including those with rather small population sizes. Populations are simulated to test the genetic gain that can be attained in a dairy trait with $\mathrm{OC}$ selection compared with truncation selection, and under different conditions, including number of young bulls, heritability of the selected trait, and rate of inbreeding.

\section{MATERIALS AND METHODS}

\section{Simulated Breeding Schemes}

The general simulated breeding scheme (Figure 1) implies selection of sires of sires (SS) and dams of sires (DS) for a dairy trait, without progeny testing. Young sires (YS) were used at the age of $1 \mathrm{yr}$ for a single year of mating with dams of dams (DD). The best YS were selected as SS. Two SS schemes were compared in the current study. In the first scheme, bulls were used as SS for just a single year. In the second scheme, SS could be used for up to $10 \mathrm{yr}$. Dams of sires were selected among all females of reproduction age. Selection of SS and DS was performed simultaneously with optimum contribution selection (OCS) or alternatively independently with TS. For female replacements, 2 schemes

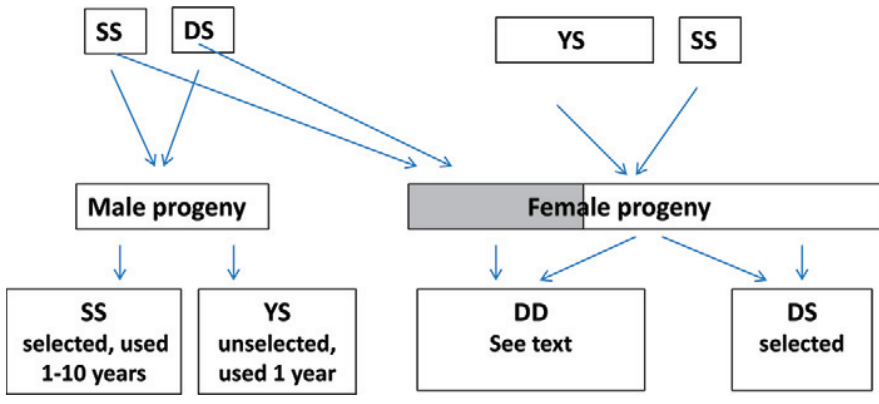

Figure 1. Simulated breeding scheme. SS = sires of sires; YS = young bulls; $\mathrm{DS}=$ dams of sires; $\mathrm{DD}=$ dams of dams. Color version available in the online PDF.

were compared. In the first (DD-Sel), female progeny born from SS per DS mating were first taken and then replacements were randomly selected from females born from YS per DD mating. In the second scheme (DDRan), all female replacements were randomly selected among all females born. The young bull scheme was used instead of the classical commercial progeny test programs because the range of population size simulated does not allow efficient progeny test of sires and because of its greater simplicity in implementation. When SS were used from 5 to $10 \mathrm{yr}$ of age, their EBV take into account their daughters' lactations even in the absence of a progeny test scheme.

\section{Simulation Model}

Age-structured populations between 500 and 6,000 adult females (FF) were stochastically simulated. Females produced 1 offspring per year from 2 to $9 \mathrm{yr}$ of age. Table 1 summarizes male and female demographic parameters of the simulated populations, including random culling rates determining age class distribution. Population age distribution and total size were not allowed to vary stochastically. Simulation interval is 1 yr, corresponding to both parturition and birth-age of reproduction intervals.

A single trait repeatability model was used to estimate animal model BLUP EBV, with a random genetic effect, environmental effects, and a residual. No fixed effects were simulated. The effect of inbreeding on the Mendelian sampling effect was ignored. A dairy trait was simulated, with a base heritability of 0.3 and repeatability of 0.5 (scenarios with other values of heritability ranging from 0.1 to 0.9 are also simulated). Annual inbreeding rate $(\boldsymbol{\Delta} \mathbf{F})$ and annual genetic gain $(\Delta \mathbf{G})$ were determined from average inbreeding coefficients and true breeding values of newborn females. The base population was assumed to be unrelated, their genotypes were sampled from the normal distribution 
Table 1. Demographic parameters of the simulated populations

\begin{tabular}{ll}
\hline Parameter & Value \\
\hline Age at first mating & Females $=1$ yr; young sires $=1$ yr; sires of sires $=1$ or 5 yr \\
Female fertility & 1 offspring/year, age 2 to 9 \\
Sex ratio at birth & $1: 1$ yr \\
Parturition interval & Females $=1 ;$ males $=$ unlimited \\
No. of matings per year & 1 to 10 \\
No. of years of use of males & Age $0-1=10 \% ; 1-2=0 \% ; 2-3=15 \% ; 3-4=6 \% ; 4-5=6 \% ;$ \\
Culling (random) females & $5-6=13 \% ; 6-7=31 \% ; 7-8=33 \% ; 8-9=33 \%$ \\
Culling (random) males & Age $0-1=15 \%$ \\
\hline
\end{tabular}

$\mathrm{N}(0,1)$ and genetic change was expressed in genetic SD units.

Rate of inbreeding can be regulated by controlling the relationship among breeding animals (Fernández et al., 2011). Maximization of genetic gain at a given rate of inbreeding (i.e., OCS) is implemented in this study by maximizing the function

$$
\mathbf{x}^{\prime} \mathbf{a}-\lambda \mathbf{x}^{\prime} \mathbf{A x}
$$

where $\mathbf{x}^{\prime}$ is the vector of contributions to the next generation of candidate parents; $\mathbf{a}$ is the vector of EBV of the candidates; and $\mathbf{A}$ is the relationship matrix among candidates weighted by $\boldsymbol{\lambda}$, which is the penalty assigned to average relationship among candidates relative to the first term of the equation, the breeding values of candidates. The contribution $x_{i}$ of candidate $i$ is equal to $1 / 2\left(n_{i} / N\right)$, where $n_{i}$ is the number of matings from animal $i$ and $N$ the total number of matings, and the coefficient $1 / 2$ ensures, for the maximization process, that the sum of contributions of each sex sum to 0.5. Number of possible matings per candidate sire was not restricted. The problem of maximizing genetic gain at a given inbreeding rate in the original proposition of Meuwissen (1997) was solved by using Lagrangian multipliers. An alternative method used by Berg et al. (2007) is simulated annealing (Press et al., 1989), previously proposed by Fernández et al. (2001), in managing genetic contribution for inbreeding control in nonselected populations. As in Berg et al. (2007), in the current study, the weight $\lambda$ on the second term of equation [1] is chosen by the operator, with preliminary simulations, in such a way that the desired annual rate of inbreeding is achieved, and maximization of equation [1] is performed by using an annealing algorithm (Press et al., 1989). When generations overlap, the contribution of the different generation cohorts weighted by future breeding opportunities should be taken into account (Meuwissen and Sonesson, 1998; Grundy et al., 2000). However, considering that in most of our simulations male generations do not overlap and for the sake of simplicity, in the current study, work contribution optimization assumes discrete generations.
Once parents and their contributions have been determined, different mating strategies can be used. The mating system usually has less influence on long-term inbreeding rate than do genetic contributions (Fernández et al.,2001); however, it has been shown that mating of parents with minimum coancestry is advantageous in optimum contribution selection (Sonesson and Meuwissen, 2000). Considering that control over mating in local breeds is generally difficult to achieve, this study uses random mating among both selected $(\mathrm{SS} \times \mathrm{SD})$ and unselected animals $(\mathrm{YS} \times \mathrm{DD})$, but avoids close inbreeding mating (i.e., between sibs and between parents and their progeny). Optimum contribution selection is compared with truncation selection on EBV at the same year inbreeding rate.

The number of DS was defined by the operator to have the desired number of YS, accounting for sex ratio at birth and random culling. The number of SS was chosen by the OCS algorithm, driven by the $\lambda$ weight and to achieve the desired inbreeding rate. In TS, the number of SS was reached by trial and error to achieve the desired inbreeding rate.

Selection was simulated for 25 yr. The generation interval was expected to vary, mainly as a function of the number of years of use of sires, from approximately 3.5 to $4.5 \mathrm{yr}$, corresponding to 7 to 5.5 generations of simulation, respectively. During the first 5 yr of simulation, TS was consistently used (with 8 SS) to build pedigrees used during the following $20 \mathrm{yr}$ of OCS. Eight hundred iterations were used. Figure 2 shows the typical dynamics of genetic level and inbreeding during the $25 \mathrm{yr}$ of selection, as the average of the 800 iterations. From yr 1 to 5, response to selection was minimal. Only after yr 15 as noise negligible and cumulated inbreeding and genetic level were monotonic and linear. Both $\Delta \mathrm{G}$ and $\Delta \mathrm{F}$ were computed at yr 25 minus level at yr 15 , divided by 10 , assuming linearity.

\section{Scenarios Simulated}

A total of 57 populations were simulated to emulate a wide range of situations that can be observed in local dairy cattle breeds. If not specified, the following base 
Table 2. Parameter values of the simulated populations with the 2 selection strategies, optimum contribution selection (OCS) and truncation selection (TS)

\begin{tabular}{lll}
\hline Parameter & OCS & TS \\
\hline Female population size & 500 to 6,000 & 500 to 6,000 \\
No. of young sires & $8,16,24,32,40$ & $8,16,32$ \\
Heritability & $0.1,0.3,0.7,0.9$ & $0.1,0.3,0.7,0.9$ \\
Inbreeding rate $(\Delta \mathrm{F} ; \%)$ & $0.1,0.2,0.3,0.4,0.5,0.6$ & $0.1,0.2,0.3,0.4,0.5,0.6$ \\
Minimum and maximum age & $1-1,1-2,1-3,1-4,1-5$, & $1-1,1-2,1-3,1-4,1-5$, \\
of sires of sires & $1-10,5-5,5-10$ & $1-10,5-5,5-10$ \\
\hline
\end{tabular}

parameters were used: heritability of 0.3 (repeatability 0.5 ), an annual inbreeding rate of $0.3 \%$, the use of SS for $1 \mathrm{yr}$ only at $1 \mathrm{yr}$ of age, and female renewal with the DD-Sel strategy. Optimum contribution selection was simulated in populations from 500 to 6,000 females and compared, at the same inbreeding rate, to TS. In both cases, 16 YS were used. Genetic gain is expected to vary as a function not only of female population size, but also according to the number of YS. The effect of the number of YS was analyzed in populations of 1,000 $\mathrm{FF}$, with YS varying from 8 to 40 , and comparing the DD-Sel and DD-Ran strategies for DD replacement. The influence of the age of $\mathrm{SS}$ on $\Delta \mathrm{G}$ was analyzed in populations of $1,000 \mathrm{FF}$, with 8 and $32 \mathrm{YS}$, with a minimum age of $1 \mathrm{yr}$ and a maximum age of 1 to $10 \mathrm{yr}$, or a minimum age of $5 \mathrm{yr}$ and a maximum age of 5 or 10 yr. Both OCS and TS were compared, at the same inbreeding rate and at different heritabilities (0.1-0.9) in populations of $500 \mathrm{FF}$ with $32 \mathrm{YS}$. Both OCS and TS were also compared at annual rates of inbreeding

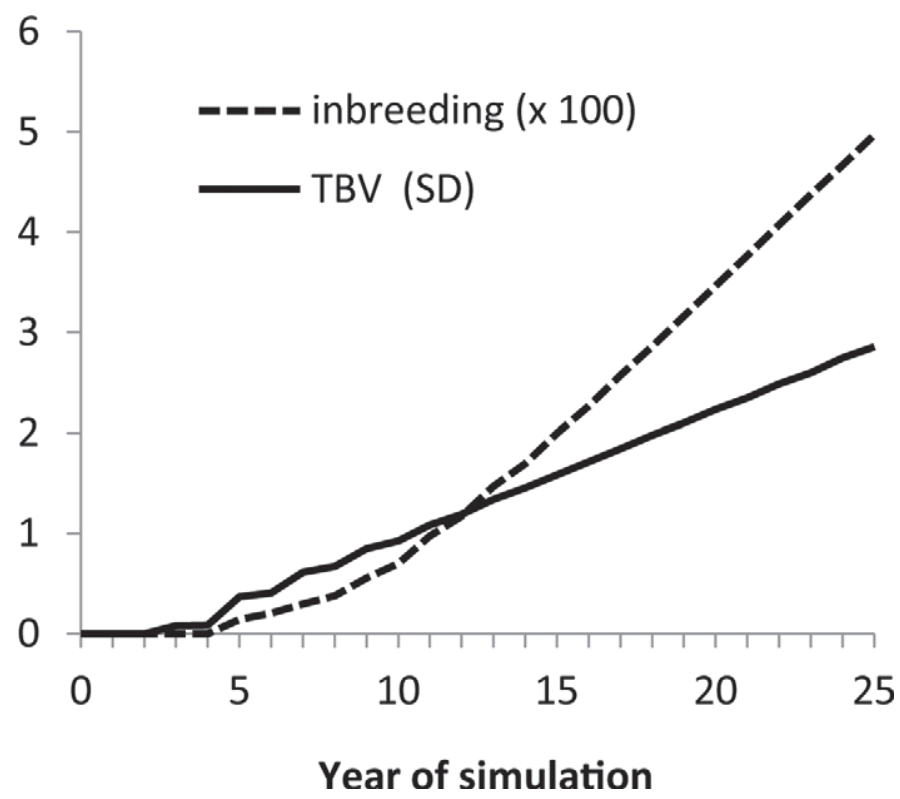

Figure 2. Cumulated genetic response, as average true breeding value (TBV), in standard deviation units (solid line), and average inbreeding $(\times 100$; dotted line) over years of simulation. different from the base value of $0.3 \%(0.1-0.6 \%)$ in populations of $500 \mathrm{FF}$ with $32 \mathrm{YS}$. Parameter values of the simulated populations are summarized in Table 2.

\section{RESULTS}

The base constraint on the annual rate of inbreeding of 0.003 holds, within a range of 0.00295 to 0.00305 , in all the simulated populations. Standard errors of both $\Delta \mathrm{F}$ and $\Delta \mathrm{G}$ were $\leq 0.001$ across all simulations.

At a fixed number of YS and DS, enlarging population size increases the selection intensity of DS and $\Delta \mathrm{G}$ were expected to increase. Figure 3 reports genetic gain in populations from 500 to $6,000 \mathrm{FF}$, with $16 \mathrm{YS}$ and all base parameters. With OCS, annual genetic gain increased rapidly as FF increases, from 500 to 4,000 (from 0.118 to 0.150 ). Then, $\Delta \mathrm{G}$ increased more slowly up to 6,000 females (0.155). The OCS was compared with TS with 16 YS and all base parameters. With TS, the annual inbreeding rate of 0.003 was achieved by setting several SS, depending on population size, of 8 (500 FF), 7 (1,000 FF), and $6(2,000-6,000 \mathrm{FF})$. At the same inbreeding rate of 0.003 , OCS produced higher genetic gain than TS. Differences in $\Delta \mathrm{G}$ between OCS and TS decreased progressively by increasing population size from $6.3(500 \mathrm{FF})$, to $3.4(4,000 \mathrm{FF})$, to $2 \%(6,000 \mathrm{FF})$. The OCS used on average, across all population sizes simulated, 0.3 SS (0.2-0.4) less than TS. The generation interval in DD was $4.6 \mathrm{yr}$ (all schemes) and was slightly shorter in DS (4.5 and 4.3 with OCS and TS, respectively). Considering that generation interval of SS and YS was constrained to $2 \mathrm{yr}$, the average generation interval was 3.3.and 3.2 yr for OCS and TS, respectively, corresponding to an inbreeding rate per generation of about $1 \%$.

The differences in genetic gain between the 2 simulated strategies for DD replacement, DD-Sel and DDRan, are reported in Table 3 in populations of 1,000 FF with several YS varying from 8 to 40 . With 8 YS, genetic gain is similar with both DD replacement strategies, 0.118 (DD-Ran) and 0.121 (DD-Sel). When we increased the number of YS, the DD-Ran genetic gain remained at the level of about 0.118; conversely, for DD-Sel, $\Delta \mathrm{G}$ increased progressively to 0.139 (40 YS), 


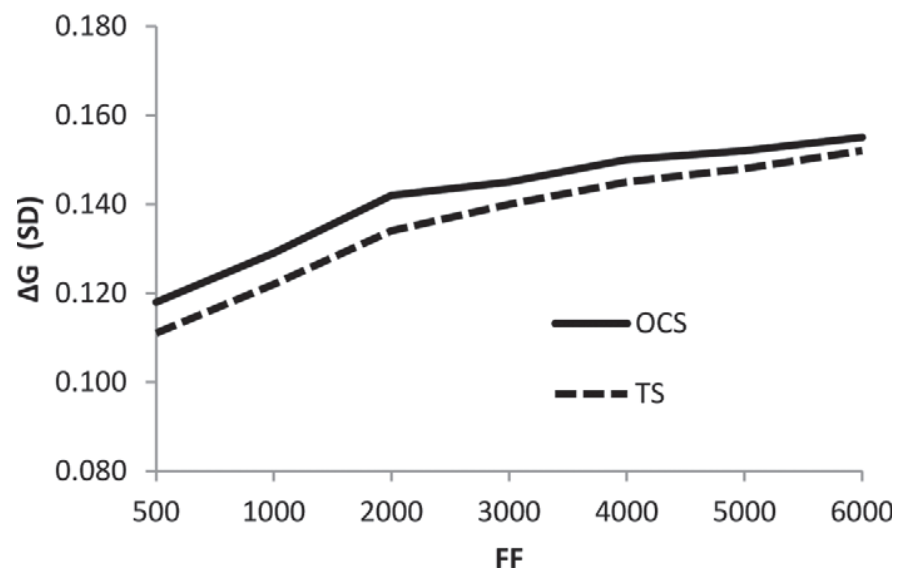

Figure 3. Annual genetic gain ( $\Delta \mathrm{G}$; standard deviation units) with optimum contribution selection (OCS) and truncation selection (TS) in populations from 500 to 6,000 females $(\mathrm{FF})$, with 16 young sires.

$17 \%$ higher than the DD-Ran strategy. The number of SS was higher with DD-Sel, with a difference that increased by raising the number of YS, from 4.5 and 4.2 (8 YS) to 9.5 and 6.0 (40 YS), respectively, in DD-Sel and DD-Ran. Average generation interval was rather constant across the $2 \mathrm{DD}$ replacement systems, and varied from $3.3(8,16 \mathrm{YS})$ to $3.2 \mathrm{yr}(24,32,40 \mathrm{YS})$.

The effects of the age of SS on genetic gain with OCS selection is analyzed in Figure 4 and Table 4 in populations of $1,000 \mathrm{FF}$ with 8 or $32 \mathrm{YS}$ and all base parameters. By keeping the minimum age of SS at 1 yr and increasing the maximum age from 1 to $10, \Delta \mathrm{G}$ increased from 0.122 to 0.135 and from 0.132 to 0.146 , respectively, for 8 and 32 YS. When minimum age of SS was set to $5 \mathrm{yr}$, genetic gain diminishes, in particular with respect to the case where SS age ranged from 1 to $10 \mathrm{yr}$. The number of SS, by changing the range of age of SS, varied from 2.3 (age 5-5) to 6.2 (age 1-5) with 8 YS, and from 3.8 (age 5-5) to 13.4 (age 1-4) with 32 YS. Generation interval of SS increased, as expected, by increasing both their minimum and maximum age, and, consequently, average population generation interval went from 3.3 (age 1-1) to $4.75 \mathrm{yr}$ (age 5-10) for $8 \mathrm{YS}$, and from 3.2 (age 1-1) to $4.5 \mathrm{yr}$ (age 5-10) for $32 \mathrm{YS}$.

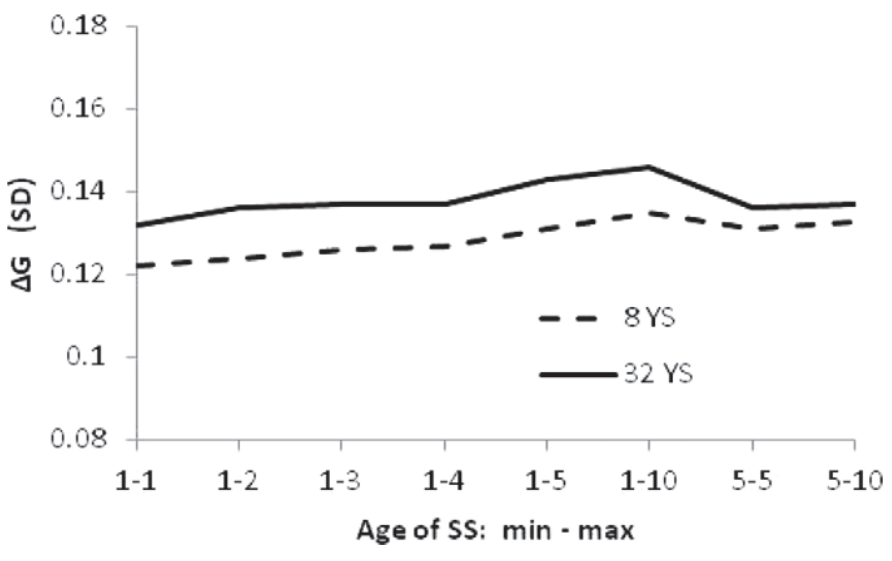

Figure 4. Genetic gain per year ( $\Delta \mathrm{G}$; standard deviation units) as the function of the age of sires of sires (SS) with optimum contribution selection in a population of 1,000 females and 8 or 32 young sires (YS).

In Table 5, annual genetic gain in populations of 500 FF with 32 YS selected for a trait with heritability of $0.1,0.3,0.7$, and 0.9 with OCS and TS selection is shown. In our simulation model, with TS selection, inbreeding rate was controlled by setting the number of SS, and therefore the desired $\Delta \mathrm{F}$ could not always be exactly achieved. Then, to compare the 2 selection methods at the same annual inbreeding rate, $\Delta \mathrm{F}$ was allowed to vary from a minimum of $0.0027\left(\mathrm{~h}^{2}=0.9\right)$ to a maximum of $0.0032\left(\mathrm{~h}^{2}=0.1\right)$. As expected, by increasing heritability, $\Delta \mathrm{G}$ increased from 0.089 and $0.086\left(\mathrm{~h}^{2}=0.1\right)$ to 0.177 and $0.164\left(\mathrm{~h}^{2}=0.9\right)$ with OCS and TS selection, respectively. We also observed that, by increasing heritability, the difference in $\Delta \mathrm{G}$ between OCS and TS selection increased from $3 \%\left(\mathrm{~h}^{2}=0.1\right.$ and 0.3 , respectively), to $5 \%\left(\mathrm{~h}^{2}=0.7\right)$, to a maximum of $8 \%\left(\mathrm{~h}^{2}=0.9\right)$ in favor of OCS. The number of SS (Table 5 ) is rather constant among the simulated populations, ranging from 9.4 to 10.1 for OCS selection and from 9.7 to 9.8 for TS selection. Average generation interval was also constant across simulated populations, ranging from 3.1 to $3.2 \mathrm{yr}$.

Table 6 compares annual genetic gain in populations of $500 \mathrm{FF}$ with $32 \mathrm{YS}$, alternatively with OCS or TS,

Table 3. Annual genetic gain $(\Delta G)$, in standard deviation units, and number of sires of sires (SS) as a function of dams of dams (DD) replacement strategy (DD-Sel and DD-Ran) and number of young sires (YS) in populations of 1,000 females with optimum contribution selection

\begin{tabular}{lcccccc}
\hline & \multicolumn{2}{c}{ DD-Sel } & & \multicolumn{2}{c}{ DD-Ran } & \\
\cline { 2 - 3 } YS & SS $(\mathrm{n})$ & $\Delta \mathrm{G}$ & & $\mathrm{SS}(\mathrm{n})$ & $\Delta \mathrm{G}$ & $\left(\Delta \mathrm{G}_{\text {DD-Sel }}-\Delta \mathrm{G}_{\text {DD-Ran }}\right) / \Delta \mathrm{G}_{\text {DD-Ran }}$ \\
\hline 8 & 4.5 & 0.121 & & 4.2 & 0.118 & 0.025 \\
16 & 6.1 & 0.129 & & 4.8 & 0.118 & 0.093 \\
24 & 7.3 & 0.132 & & 5.4 & 0.119 & 0.109 \\
32 & 8.4 & 0.136 & & 5.8 & 0.119 & 0.143 \\
40 & 9.5 & 0.139 & & 6.0 & 0.119 & 0.168 \\
\hline
\end{tabular}


Table 4. Effects of the age of sires of sires (SS) with optimum contribution selection on genetic gain per year $(\Delta \mathrm{G})$, in standard deviation units, number of SS, generation intervals (Gen Int.), with population sizes of 1,000 females and 8 or 32 young sires (YS)

\begin{tabular}{|c|c|c|c|c|c|c|c|c|}
\hline \multirow{2}{*}{$\begin{array}{l}\text { Age of SS } \\
\text { (minimum-- } \\
\text { maximum) }\end{array}$} & \multicolumn{4}{|c|}{$8 \mathrm{YS}$} & \multicolumn{4}{|c|}{$32 \mathrm{YS}$} \\
\hline & $\Delta \mathrm{G}$ & $\mathrm{SS}(\mathrm{n})$ & $\begin{array}{l}\text { Gen Int. } \\
\quad(\mathrm{SS})\end{array}$ & $\begin{array}{c}\text { Gen Int. } \\
\text { (mean) }\end{array}$ & $\Delta \mathrm{G}$ & $\mathrm{SS}(\mathrm{n})$ & $\begin{array}{l}\text { Gen Int. } \\
\text { (SS) }\end{array}$ & $\begin{array}{l}\text { Gen Int } \\
\text { (mean) }\end{array}$ \\
\hline $1-1$ & 0.122 & 4.5 & 2 & 3.30 & 0.132 & 8.6 & 2 & 3.15 \\
\hline $1-2$ & 0.124 & 5.5 & 2.4 & 3.40 & 0.136 & 11.7 & 2.4 & 3.25 \\
\hline $1-3$ & 0.126 & 5.9 & 2.7 & 3.50 & 0.137 & 13.0 & 2.6 & 3.30 \\
\hline $1-4$ & 0.127 & 6.1 & 2.9 & 3.57 & 0.137 & 13.4 & 2.8 & 3.35 \\
\hline $1-5$ & 0.131 & 6.2 & 3.7 & 3.75 & 0.143 & 12.4 & 3.6 & 3.53 \\
\hline $1-10$ & 0.135 & 6 & 5.6 & 4.23 & 0.146 & 12.3 & 5.1 & 3.88 \\
\hline $5-5$ & 0.131 & 2.3 & 6 & 4.20 & 0.136 & 3.8 & 6 & 4.08 \\
\hline $5-10$ & 0.133 & 4.7 & 7.7 & 4.75 & 0.137 & 8.6 & 7.5 & 4.50 \\
\hline
\end{tabular}

with different constraint values on the annual rate of inbreeding, from 0.1 to $0.6 \%$. As expected, by increasing the constraint on inbreeding, $\Delta \mathrm{G}$ decreased from 0.138 and $0.134(\Delta \mathrm{F}=0.6 \%)$ to 0.097 and $0.081(\Delta \mathrm{F}$ $=0.1 \%$ ) in OCS and TS populations, respectively. By relaxing the constraint on $\Delta \mathrm{F}$ from 1 to $4 \%$, the difference of $\Delta \mathrm{G}$ between OCS and TS increased. The advantage of OCS at a $\Delta \mathrm{F}$ of $0.1 \%$ is almost $20 \%$, but it dropped to $6 \%$ at $\Delta \mathrm{F}$ of $0.2 \%$, to $3 \%$ at $\Delta \mathrm{F} 0.4 \%$, and thereafter remained rather constant. The number of SS (Table 6) ranged from 4.2 and 5 at $0.6 \% \Delta \mathrm{F}$, and increased to 21.4 and 20.2 at $0.1 \% \Delta \mathrm{F}$ in OCS and TS populations, respectively. Average generation interval was constant at an average value of 3.2 across all simulated populations.

\section{DISCUSSION}

In local breeds focus is usually on pure management of genetic diversity and inbreeding control. However, selection is an appropriate tool to improve performance in quality and production traits, in the aim of promoting not only conservation of local breeds but also their sustainable use (FAO, 2007). In this direction, selection strategies need to attentively consider possible negative effects on those traits determining the conservation values and on key adaptation traits; these might include adaption to a harsh environment or to low-input production systems or traits, such as longevity, fertility, and quality of meat and milk (Gandini and Oldenbroek, 2007).

The inbreeding rate per generation of about 0.01 simulated in the base scenario is within the range recommended by the international guidelines (FAO, 2013). When SS are used for more than a single year, a maximum generation interval of $4.75 \mathrm{yr}$ is observed, corresponding to a generation inbreeding rate of 0.0014 , somewhat above the recommended rates. In any case, we stress that populations with different generation intervals are compared in the current paper at the same inbreeding rate per year. In large, simulated populations, traditional progeny test schemes can attain (with a trait heritability of 0.4 ) 0.22 genetic SD of genetic response per year (Schaeffer, 2006); the current study shows that reasonable genetic gains (i.e., about 50 to $70 \%$ of that achievable in large populations) can be achieved at a recommended inbreeding rate in populations of only 500 to 6,000 females.

Considering a trait with a heritability of 0.3 and inbreeding rate constrained to 0.003 per year, genetic gain per year is 0.118 with 500 females and increases to 0.155 with 6,000 females. The population sizes simulated in this study cover the range of population sizes of the local dairy cattle breeds designated as endangered

Table 5. Annual genetic gain $(\Delta \mathrm{G})$, in standard deviation units, annual inbreeding rate $(\Delta \mathrm{F})$, and number of sires of sires (SS) as a function of the heritability of the selected trait, with optimum contribution (OCS) or truncation (TS) selection ${ }^{1}$

\begin{tabular}{|c|c|c|c|c|c|c|c|}
\hline \multirow[b]{2}{*}{ Heritability } & \multicolumn{3}{|c|}{ OCS } & \multicolumn{3}{|c|}{ TS } & \multirow[b]{2}{*}{$\left(\Delta \mathrm{G}_{\mathrm{OCS}}-\Delta \mathrm{G}_{\mathrm{TS}}\right) / \Delta \mathrm{G}_{\mathrm{TS}}$} \\
\hline & SS (n) & $\Delta \mathrm{G}$ & $\Delta \mathrm{F}$ & SS (n) & $\Delta \mathrm{G}$ & $\Delta \mathrm{F}$ & \\
\hline 0.1 & 9.4 & 0.089 & 0.322 & 9.7 & 0.086 & 0.322 & 0.035 \\
\hline 0.3 & 9.8 & 0.124 & 0.299 & 9.8 & 0.120 & 0.302 & 0.033 \\
\hline 0.7 & 9.8 & 0.162 & 0.279 & 9.8 & 0.154 & 0.279 & 0.052 \\
\hline 0.9 & 10.1 & 0.177 & 0.272 & 9.8 & 0.164 & 0.273 & 0.079 \\
\hline
\end{tabular}

${ }^{1}$ Population size is 500 females and the number of young sires is 32 . 
Table 6. Annual genetic gain $(\Delta \mathrm{G})$, in standard deviation units, and number of sires of sires (SS) at different annual rates of inbreeding $(\Delta \mathrm{F})$, with optimum contribution (OCS) or truncation (TS) selection ${ }^{1}$

\begin{tabular}{|c|c|c|c|c|c|}
\hline \multirow[b]{2}{*}{$\Delta \mathrm{F}(\%)$} & \multicolumn{2}{|c|}{ OCS } & \multicolumn{2}{|c|}{ TS } & \multirow[b]{2}{*}{$\left(\Delta \mathrm{G}_{\mathrm{OCS}}-\Delta \mathrm{G}_{\mathrm{TS}}\right) / \Delta \mathrm{G}_{\mathrm{TS}}$} \\
\hline & SS (n) & $\Delta \mathrm{G}$ & SS (n) & $\Delta \mathrm{G}$ & \\
\hline 0.1 & 20.2 & 0.097 & 21.4 & 0.081 & 0.198 \\
\hline 0.2 & 13.6 & 0.114 & 14.35 & 0.108 & 0.056 \\
\hline 0.3 & 9.8 & 0.124 & 9.8 & 0.120 & 0.033 \\
\hline 0.4 & 7.5 & 0.131 & 7.0 & 0.127 & 0.031 \\
\hline 0.5 & 6.1 & 0.136 & 5.0 & 0.130 & 0.031 \\
\hline 0.6 & 5.0 & 0.138 & 4.2 & 0.134 & 0.030 \\
\hline
\end{tabular}

${ }^{1}$ Population size is 500 females and the number of young sires is 32 .

according to criteria of the European Commission (Gandini et al., 2005). Figure 3 shows that the majority of the differences in genetic gain as a function of recorded population are observed between populations of 500 versus 4,000 females. Relatively little difference was observed between populations of 4,000 and 6,000.

The OCS produces higher genetic gain than TS, with differences ranging from 2 to $6.3 \%$. In simulated populations with overlapping generations, Meuwissen and Sonesson (1998) observed advantages in genetic gain of OCS ranging from 16 to $44 \%$ relative to TS. The advantage of OCS versus TS was higher at smaller population sizes, as observed in our study. The authors suggest that the large differences in genetic gain observed in favor of OCS could partially be due to the lack of optimization of their TS scheme. In addition, we can argue that the higher advantage observed by Meuwissen and Sonesson (1998) may be associated with the higher selection intensity and index accuracy used, and in particular to the fact that, to achieve the optimal contributions among females, in their selection schemes dams were assumed to produce an unlimited number of offspring, a unrealistic situation in cattle populations. Concerning index accuracy, Meuwissen and Sonesson (1998) reported higher advantages of OCS versus TS in progeny-tested versus non-progeny-tested populations.

The potential of OCS has also been analyzed in very large livestock populations by comparing optimized retrospective selection with the observed values in one step of selection with constraints on inbreeding. In Meatlinc sheep and Aberdeen Angus cattle, Avendaño et al. (2003) found up to $30 \%$ more genetic gain than with TS. In the UK Holstein population, Kearney et al. (2004) also observed higher genetic response when using optimized contributions versus observed contributions. Koenig and Simianer (2006), by mimicking the next generation of German Holstein AI bulls, observed $13 \%$ more genetic gain compared with the actual breeding program under the same average relationship constraint. In these cited studies, OCS possibly detected that rather unbalanced use of ancestors occurred in populations due to their past management with no inbreeding control, with larger advantages of OCS versus TS, as suggested by Fernández et al., (2011). Gourdine et al. (2012) simulated selection progress in local pig breeds of 300 to 1,848 females and compared OCS inbreeding rates to TS rates. Those authors showed that, at a similar genetic gain, inbreeding can be considerably lower with OCS, up to $80 \%$, but results are not comparable with those of our study because of the different breeding scheme used.

The effects of varying the number of young sires, and consequently the number of dams of sires, are analyzed with the 2 strategies of female replacement, DD-Ran and DD-Sel (Table 3). With no selection on the pathway DD, genetic gain per year remains constant in the range of the number of YS simulated (8-40). This occurs because the higher selection intensity among SS induced by increasing the number of YS is compensated by the smaller selection intensity in the pathway DS, and in general because SS selection is penalized by the low accurate pedigree indices. The OCS makes use of the larger number of candidates available when the number of YS is increased by selecting higher numbers of SS, which increases from 4.5 for 8 YS to 9.5 for 40 YS. Increasing the number of YS produces consistently higher genetic gain when the females born from SS per DS are first kept for female replacement. It should be noted that an increase of genetic gain from the DD pathway could be also obtained by selecting DD after selection of DS, thus avoiding costs associated to raising high numbers of YS. Finally, the fact that the number of SS in the DD-Sel strategy is higher than in the DD-Ran strategy could be due to the fact that the number of dams decreases in DD-Sel with respect to DD-Ran, and higher numbers of SS are necessary to respect the inbreeding constraint.

Increasing the number of years of use of SS implies costs and organization of semen preservation; however, it has beneficial effects on genetic gain (Figure 4 and Table 4). By increasing the number of years of use of SS, we enlarge the number of candidates and probably 
their variability in terms of both genetic relationship and EBV. Optimum contribution selection uses the wider range of candidates, generation interval increases, and in all simulated cases at least $50 \%$ of the SS are mated at an age higher than 1 . The EBV of SS of 5 yr and more have a higher accuracy of being based on daughter lactations, even if a progeny testing is not planned. However, OCS selection cannot take this aspect into account, and forcing the age of SS to be $5 \mathrm{yr}$ or more does not produce benefits in genetic gain.

When comparing OCS versus TS at different heritabilities, we observed an advantage of OCS by increasing heritability above 0.3. Brisbane and Gibson (1995) and Meuwissen (1997) observed an opposite trend, expected by the fact that EBV are more highly correlated, the effect of selection on inbreeding is larger, and OCS might find animals of high additive genetic merit in families less related when heritability is lower. Our results are most probably due to the low accuracy of EBV of the young bull scheme used; by increasing heritability the accuracy of pedigree index increased and we observed OCS to be working more efficiently. In this regard, as previously mentioned, Meuwissen and Sonesson (1998) report an advantage of OCS over TS with progenytested candidates opposite to candidates evaluated on pedigree index.

Meuwissen and Woolliams (1994) balanced the drift of current deleterious mutations against natural selection and recommended for livestock populations an effective size of 50 or above, corresponding to a generation inbreeding rate $<1 \%$. Most simulations in this study were done within this range. However, higher or smaller inbreeding rates might be acceptable under specific conditions and as a function of the selection time period. More generally, when acceptable rates of inbreeding are not clear, it has been suggested to generate a response surface of inbreeding versus genetic gain to orient the choice of the inbreeding level to be adopted (Brisbane and Gibson, 1995; Meuwissen, 1997). In our work, by increasing the constraint on inbreeding, the difference of genetic gain between the 2 selection methods increases in favor of OCS, and the advantage at the lowest inbreeding level simulated of $0.1 \%$ is almost 6 times higher than at the base inbreeding rate of $0.3 \%$. Meuwissen (1997) and Meuwissen and Sonesson (1998) have also noticed that the superiority of the optimal contribution method increases when smaller rates of inbreeding are requested. At high inbreeding rates, the cost $(\lambda)$ on relationship (equation [1]) is low and the search for optimal contributions is limited, null at $\lambda$ equal to zero. At low inbreeding rates, however, the optimal contribution selection looks for parents to maximize genetic gain at the given inbreeding constraint and the 2 selection methods differ more in their solutions. An opposite trend was reported by Villanueva et al. (2006), with an increase of the advantage of optimum selection versus TS when decreasing the constraint on inbreeding, explained by changes in family structures when increasing the size of the scheme. More generally, we can expect that population structure and distribution of EBV or genetic relationships of candidates affect the superiority of optimum selection versus truncation selection, together with the constraint on rate of inbreeding adopted.

\section{CONCLUSIONS}

The main objective of this work is to understand the rate of genetic gain that can be attained, with an acceptable inbreeding rate, in local dairy cattle breeds from 500 to 6,000 females, where selection is generally disregarded for its effect in accelerating excessive inbreeding. Besides population size, the effects of the number of sires, of the heritability of the selected trait, and of the level of constraint on inbreeding rates are investigated. Results show that opportunities exist for selection, even in populations of a few hundred or thousand females. A young bull selection scheme was used because its simplicity in field implementation. Optimum contribution selection at the low selection intensities of the simulated schemes has not shown much higher genetic gains than TS. However, OCS remains an advisable tool to control inbreeding in selection schemes where expected effective population sizes, and consequently expected inbreeding rates, are difficult to estimate. Selection schemes should be adapted to the low or medium input production systems of local breeds, and should be easy to implement at reasonable costs. Implementation of selection in local breeds will most often require specific investments in infrastructure and manpower considering, among other factors, the need for accurate data recording and selection skills and the presence of AI and breeders organizations. A cost-benefit analysis is therefore advisable before considering the implementation of selection schemes in local dairy cattle breeds. Some costs are expected to be high; for example, if we consider that a bull will produce a few doses of semen with costs per unit much higher than in larger breeds. A benefit analysis should include, besides the higher expected production per animal unit, the opportunity to make the local breed more attractive to farmers, and as such contribute to the conservation of farm animal diversity.

\section{ACKNOWLEDGMENTS}

This work was supported by the SelMol project (MiPAAF, Rome, Italy) and by the Accordo Quadro 
CNR-Regione Lombardia (Palazzo Lombardia, Milan, Italy), project "Risorse Biologiche e Tecnologie per lo Sviluppo Sostenibile del Sistema Agro-alimentare, WP4."

\section{REFERENCES}

Avendaño, S., B. Villanueva, and J. A. Woolliams. 2003. Expected increases in genetic merit from using optimized contributions in two livestock populations of beef cattle and sheep. J. Anim. Sci. 81:2964-2975.

Berg, P., M. K. Sørensen, and J. Nielsen. 2007. Eva Interface User Manual. Accessed June 18, 2013. http://eva.agrsci.dk/index.html.

Brisbane, J. R., and J. P. Gibson. 1995. Balancing selection response and rate of inbreeding by including genetic relationship in selection decisions. Theor. Appl. Genet. 91:421-431.

European Union. 2005. Council Regulation (EU) No 1698/2005. Accessed Oct. 15, 2013. http://eur-lex.europa.eu/LexUriServ/ LexUriServ.do?uri=OJ:L:2005:277:0001:0040:EN:PDF.

FAO (Food and Agriculture Organization of the United Nations). 2007. The global strategy for the management of farm animal genetic resources and the Interlaken declaration. Accessed Jan. 5, 2014. ftp://ftp.fao.org/docrep/fao/010/a1404e/a1404e00.pdf.

FAO (Food and Agriculture Organization of the United Nations). 2013. In vivo conservation of animal genetic resources. FAO Animal Production and Health Guidelines. No. 14. FAO, Rome, Italy.

Fernández, J., T. H. E. Meuwissen, M. A. Toro, and A. Maki Tanila. 2011. Management of genetic diversity in small farm animal populations. Animal 5:1684-1698.

Fernández, J., M. A. Toro, and A. Caballero. 2001. Practical implementation of optimal management strategies in conservation programmes: A mate selection method. Anim. Biodivers. Conserv. $24: 17-24$.

Gandini, G., and K. Oldenbroek. 2007. Strategies for moving from conservation to utilization. Pages 29-54 in Utilization and Conservation of Farm Animal Genetic Resources. K. Oldenbroek, ed. Wageningen Academic Publisher, Wageningen, the Netherlands.

Gandini, G. C., L. Ollivier, B. Danell, O. Distl, A. Georgudis, E. Groeneveld, E. Martiniuk, J. van Arendonk, and J. Woolliams. 2005. Criteria to assess the degree of endangerment of livestock breeds in Europe. Livest. Prod. Sci. 91:173-182.
Gandini, G., and E. Villa. 2003. Analysis of the cultural value of local livestock breeds: a methodology. J. Anim. Breed. Genet. 120:1-11.

Gourdine, J. L., A. C. Sorenson, and L. Rydhmer. 2012. There is room for selection in a small local pig breed when using optimum contribution selection: A simulation study. J. Anim. Sci. 90:76-84.

Grundy, B., B. Villanueva, and J. A. Woolliams. 1998. Dynamic selection procedures for constrained inbreeding and their consequences for pedigree development. Genet. Res. 72:159-168.

Grundy, B., B. Villanueva, and J. A. Woolliams. 2000. Dynamic selection for maximizing response with constrained inbreeding in schemes with overlapping generations. Anim. Sci. 70:373-382.

Hinrichs, D., and T. H. E. Meuwissen. 2011. Analysing the effect of different approaches of penalized relationship in multi stage selection schemes. J. Anim. Sci. 89:3426-3432

Kearney, J. F., E. Wall, B. Villanueva, and M. P. Coffey. 2004. Inbreeding trends and application of optimized selection in the UK Holstein population. J. Dairy Sci. 87:3503-3509.

Koenig, S., and H. Simianer. 2006. Approaches to the management of inbreeding and relationship in the German Holstein dairy cattle population. Livest. Sci. 103:40-53.

Meuwissen, T. H. E. 1997. Maximizing the response of selection with predefined rate of inbreeding. J. Anim. Sci. 75:934-940.

Meuwissen, T. H. E., and A. Sonesson. 1998. Maximizing the response of selection with a predefined rate of inbreeding: Overlapping generations. J. Anim. Sci. 76:2575-2583.

Meuwissen, T. H. E., and J. A. Woolliams. 1994. Effective size of livestock populations to prevent a decline in fitness. Theor. Appl. Genet. 89:1019-1026.

Press, W. H., B. P. Flannery, S. A. Teulosky, and W. T. Vetterling. 1989. Numerical Recipes. Cambridge University Press, Cambridge, UK.

Schaeffer, L.R. 2006. Strategy for applying genome-wide selection in dairy cattle. J. Anim. Breed. Genet. 123:218-223.

Sonesson, A. K., and T. H. E. Meuwissen. 2000. Mating schemes for optimum contribution selection with constrained rates of inbreeding. Genet. Sel. Evol. 32:231-248.

Sørensen, M. K., A. C. Sørensen, R. Baumung, S. Borchersenand, and P. Berg. 2008. Optimal genetic contribution selection in Danish Holstein depends on pedigree quality. Livest. Sci. 118:212-222.

Villanueva, B., S. Avendaño, and J. A. Woolliams. 2006. Prediction of genetic gain from quadratic optimization with constrained rates of inbreeding. Genet. Sel. Evol. 38:127-146. 